\title{
Wasserbau, Schifffahrt und Ökologie an der Donau - Pilotprojekt Bad Deutsch-Altenburg
}

An der Donau östlich von Wien existieren Probleme für die Schifffahrt, Ökologie und den Wasserbau. Die Schifffahrt besitzt bei Niederwasser besonders in Furtbereichen eine nicht ausreichende Fahrwassertiefe, womit kostenintensive Erhaltungsmaßnahmen wie Baggerungen einhergehen. Seit einigen Jahren wird das Baggergut im System gehalten, nach flussauf transportiert und dort wieder verklappt. Eine zentrale Problemstellung betrifft die fortschreitende Sohleintiefung der Donau in diesem Abschnitt, die mit ca. $2 \mathrm{~cm}$ pro Jahr zu einer Absenkung der Wasserspiegellagen führt. Dort wo die Mächtigkeit des alluvialen Kieskörpers nur sehr gering ist, kann es im „worst case“ zu einem Sohldurchschlag kommen, wodurch eventuell eine canyonartige Erosion in die feineren marinen Sedimente möglich wäre. Daher ist die Sohlstabilisierung von zentraler Bedeutung. Die Absenkung der Wasserspiegellagen führt in den angrenzenden Vorländern des Nationalparks Donauauen über weite Strecken zur Gefahr einer mittel- bis langfristigen Austrocknung der Auwälder. Weiters ist der ökologische Zustand durch den hohen Regulierungsgrad der Donau mit durchgehenden Ufersicherungen, Leitwerken und Buhnen, die infolge der Sohleintiefung bereits bis über Mittelwasser wirken, und durch die Abtrennung der Seitenarme beeinflusst. Daraus ergeben sich die Ziele Sohlstabilisierung, Verbesserung der Schifffahrtsverhältnisse und des ökologischen Zustandes unter Berücksichtigung des Hochwasserschutzes, die im Flussbaulichen Gesamtprojekt verfolgt wurden.

Das Pilotprojekt Bad Deutsch-Altenburg (BDA) setzte nun erstmals auf ca. $3 \mathrm{~km}$ Flussstrecke alle Maßnahmen (Granulometrische Sohlverbesserung (GSV), Buhnenoptimierung, Uferrückbau und Gewässervernetzung) gleichzeitig um und ermöglichte als Naturversuch Erkenntnisgewinn in baupraktischer Hinsicht und betreffend die gemeinsame Wirkung der Maßnahmen. Dazu wurde ein integratives, wissenschaftliches Monitoring konzipiert, das abiotische und biotische Parameter umfasst.

In dieser Ausgabe der ÖWAW werden die aus dem Pilotprojekt BDA abgeleiteten Erkenntnisse des Monitorings vor, während und nach der Bauumsetzung in acht Artikeln betreffend Schifffahrt, Ökologie und Wasserbau dargestellt. Dabei ist zu erwähnen, dass das Monitoring nach Bau fortgesetzt wird und die Artikel daher die ersten Erkenntnisse zusammenfassen.

Der erste Artikel „Pilotprojekt Bad Deutsch-Altenburg - Projektmotivation, Maßnahmen, Prozessbeteiligung" von Tögel und Baumgartner erläutert aus der Sicht von viadonau als Projektträger und Nationalpark Donauauen das Pilotprojekt BDA. Nach der Beschreibung der Problemstellungen an der Donau östlich von Wien werden die drei Ziele Sohlstabilität, Verbesserungen für die Ökologie und Verbesserungen für die Schifffahrt angeführt. Dann folgt die Erläuterung der Ziele und Maßnahmen des Pilotprojektes mit der GSV, der Optimierung der Niederwasserregulierung, dem Uferrückbau und der Gewässervernetzung Johler Arm. Der Artikel schließt mit dem Beteiligungsmodell, das ein Akteursforum und Science Board beinhaltete.

Die zentrale Problemstellung der Sohleintiefung an der Donau östlich von Wien behandelt der Beitrag „Ausmaß und Dynamik der Sohlerosion der Donau östlich von Wien, flussmorphologische und wasserwirtschaftliche Aspekte“ (Klasz et al.). In der langfristigen Betrachtung zeigen Klasz et al., dass an der Donau östlich von Wien erst ab Mitte des 20. Jahrhunderts Sohllagen und Nieder- und Mittelwassersstände stark absanken, wobei sich dieser Trend - verstärkt durch den Kraftwerksausbau auch heute trotz ständiger Geschiebezugaben zur Sicherung des Unterwassers am Kraftwerk WienFreudenau fortsetzt. Das aus der Strecke ausgetragene Material wird im Stauraum des Kraftwerkes Gabčikovo abgelagert. 
Die im Zuge des wissenschaftlichen Monitorings gewonnenen Erkenntnisse zu „Grundlegenden Prozessen betreffend Hydraulik, Sedimenttransport und Flussmorphologie an der Donau“ behandeln Gmeiner et al.. Für die Untersuchungen im Rahmen des CD-Labors „IM_Fluss“ wurden innovative Monitoring- und Modellierungsansätze (z. B. Sohlschubspannungsmessgerät, 3D-Feststofftransportmodell) entwickelt. Die Erkenntnisse reichen vom Geschiebetransport mit im Vergleich zur Theorie nahezu durchgehender Bewegung beginnend bei Niederwasser und sehr hohen Transportraten, z. B. beim Hochwasser 2013, bis zu erstmals an der Donau dokumentierten, sich sehr rasch bewegenden Kiesdünen.

Die „Funktionalität der getesteten flussbaulichen Maßnahmen im Pilotprojekt Bad Deutsch-Altenburg“ analysieren Liedermann et al.. Die Wirksamkeit der GSV war in Bezug auf die Eintiefungstendenz der Donau weniger ausgeprägt als im Zuge der Planung und der vorangegangenen Modellversuche erwartet, die größeren Korngrößen konnten allerdings einen nennenswerten Beitrag leisten. Die Auswirkung der Buhnenumbauten war hingegen stärker als angenommen und führte in Teilbereichen sogar zu einer Trendumkehr im Sinne einer Auflandungstendenz, die nun eine Optimierung der Maßnahme notwendig macht. Der Uferrückbau führte zu sanfteren Ufergradienten und leistete durch den Aufweitungseffekt auch einen Beitrag zur Reduktion der Eintiefungstendenz.

Die „Auswirkungen des Pilotversuchs BDA auf die Grundwasserverhältnisse“ werden im Artikel von Derx und Blaschke behandelt. Dabei geht es um die Auswirkung der baulichen Maßnahmen auf die Bedingungen für die Austauschprozesse zwischen Grund- und Flusswasser. Gemäß Derx und Blaschke zeigten die bisherigen Analysen der Grundwasserverhältnisse vor und nach den Baumaßnahmen im Pilotversuch BDA keine wesentliche Beeinflussung des Grundwassers durch die Baumaßnahmen. Die Interaktion des Grundwassers mit der Donau blieb ebenso intakt. Das Monitoring wird aber weitergeführt, womit die Aussagekraft der Messparameter nachgewiesen werden kann und längerfristige Auswirkungen beobachtet werden können.

Der Artikel „Grundlegende Erkenntnisse im Rahmen des Pilotprojektes Bad Deutsch-Altenburg - Die Bedeutung der Stromsohle und Uferzonen für ökologische Prozesse und Artengemeinschaften an einem stark regulierten Fluss, der Donau“ von Bondar-Kunze et al. zeigt, dass Flüsse für eine langfristige ökologische Stabilität Bereiche benötigen, die bei verschiedenen Abflusssituationen als hoch produktive Lebensräume verfügbar sind und entsprechend mit dem Fluss in Austausch stehen. In diesem Artikel werden Ergebnisse von mehrjährigen Forschungsarbeiten in den Tiefenzonen der Donau und typischen Uferzonenlebensräumen für die Organismengruppen benthische Algen, Makrozoobenthos und Fische präsentiert.

Hier ist zu erwähnen, dass im Monitoringprogramm z. B. auch Vögel inkludiert sind, was im Detail den Rahmen dieses Heftes überschritten hätte.

Korner und Staudinger stellen die „qualitative und quantitative Entwicklung der Pioniervegetation an der Donau im Bereich der Pilotprojekte Bad Deutsch-Altenburg und Witzelsdorf“ dar. Flächen mit Pioniervegetation dynamischer Standorte auf Kiesflächen und Schlammbänken finden sich generell an der Donau nur noch im Anlandungsbereich von Inseln und an Gleitufern. Gemäß Korner und Staudinger wurde durch den Buhnenneubau mit abgesenkten Buhnenwurzeln, durch die Entfernung von Wasserbausteinen aus den Buhnenfeldern, durch die Schüttung einer neuen Kiesinsel im Buhnenfeld, die Herstellung eines „Hinterrinners“ im Buhnenfeld sowie durch den Uferrückbau ein wichtiger Schritt in Richtung Dynamisierung und somit größerer Naturnähe gesetzt.

Der letzte Beitrag von Habersack et al. fasst die „Erkenntnisse aus dem Pilotprojekt Bad DeutschAltenburg an der Donau für Schifffahrt, Ökologie und Wasserbau“ zusammen. Für die Schifffahrt ergaben sich vermehrte Anlandungen im Furtbereich BDA, geringere Fließgeschwindigkeiten entlang der Buhnenköpfe und etwas mehr verfügbare Fahrbreite. Betreffend die Ökologie konnte durch den Uferrückbau eine Rheophilisierung der Uferbereiche und des Johler Arms erreicht werden. Bezüg- 


\section{Editorial}

lich des Wasserbaus konnten Methoden optimiert werden, die einen Beitrag zur Sohlstabilisierung leisten (GSV und vor allem Buhnenumbauten). Eine Kombination aus Optimierung der wasserbaulichen Maßnahmen und Geschiebebewirtschaftung sollte im Sinne eines Geschiebemanagements eine nachhaltige Verbesserung der Donau östlich von Wien ermöglichen.

Das Pilotprojekt BDA findet sich zusammenfassend in einem Video auf youtube: http://youtu.be/ mAGty2Y7tdg.

\section{Univ.-Prof. DI Dr. H. Habersack ( $ه$ )}

Leiter Christian Doppler Labor

für Innovative Methoden in

Fließgewässermonitoring, Modellierung

und Flussbau, Leiter Institut für

Wasserwirtschaft, Hydrologie und konstruktiven Wasserbau, Department für Wasser - Atmosphäre - Umwelt, Universität für Bodenkultur Wien, Muthgasse 107,

1190 Wien, Österreich

E-Mail: helmut.habersack@boku.ac.at 